\title{
Grid-Enabled Non-Invasive Blood Glucose Measurement
}

\author{
Ibrahim Elsayed ${ }^{1}$, Jianguo Han ${ }^{2}$, Ting Liu ${ }^{3}$, Alexander Wöhrer ${ }^{1}$, \\ Fakhri Alam Khan ${ }^{1}$, and Peter Brezany ${ }^{1}$ \\ ${ }^{1}$ Institute of Scientific Computing \\ University of Vienna, Nordbergstrasse 15/C/3, A-1090 Vienna, Austria \\ \{elsayed, woehrer, khan, brezany\}@par.univie.ac .at \\ ${ }^{2}$ School of Information Science and Technology \\ Beijing University of Chemical Technology \\ 15 BeiSanhuan East Road, ChaoYang District, Beijing 100029, China \\ han@mail. buct.edu.cn \\ ${ }^{3}$ School of Earth and Space Science, Peking University, Beijing 100871, China \\ liuting5331@163.com
}

\begin{abstract}
Earth and life sciences are at the forefront to successfully include computational simulations and modeling. Medical applications are often mentioned as the killer applications for the Grid. The complex methodology and models of Traditional Chinese Medicine offer different approaches to diagnose and treat a persons health condition than typical Western medicine. A possibility to make this often hidden knowledge explicit and available to a broader audience will result in mutual synergies for Western and Chinese medicine as well as improved patient care. This paper proposes the design and implementation of a method to accurately estimate blood glucose values using a novel non-invasive method based on electro-transformation measures in human body meridians. The framework used for this scientific computing collaboration, namely the ChinaAustria Data Grid (CADGrid) framework, provides an Intelligence Base offering commonly used models and algorithms as Web/Grid-Services. The controlled execution of the Non-Invasive Blood Glucose Measurement Service and the management of scientific data that arise from model execution can be seen as the first application on top of the CADGrid.
\end{abstract}

Keywords: Traditional Chinese Medicine, Non-Invasive Blood Glucose Measurement, Grid Computing, e-Infrastructure.

\section{Introduction}

Grid computing [1] promises to change the way scientists will tackle future research challenges in a number of domains, including earth sciences [2], medicine 344 and life sciences [5. Service-oriented architectures (SOA) 6] are utilized in Grid computing to facilitate the virtualization of heterogeneous resources, e.g. data sources, computational resources, network and workload. Due to wireless connectivity improvements and hardware getting mobile and constantly smaller

M. Bubak et al. (Eds.): ICCS 2008, Part I, LNCS 5101, pp. 76-85, 2008.

(C) Springer-Verlag Berlin Heidelberg 2008 
and cheaper, the visions of ubiquitous [7] and mobile computing [8] are becoming reality. Information is collected, exchanged and processed by specialized computing units embedded in the environment to achieve a certain task [9] and accessed as needed on-the-way [10. Enormous amount of data will be produced at a rate never seen before in any field of human activity, requiring next generation grids 11] to cope with and making use of it.

Traditional Chinese Medicine (TCM) 12 has different models and approaches to diagnose and treat a persons health condition than typical Western medicine. According to meridian-theory, which is an important part of TCM, meridians are a secret to our biological and medical knowledge. Recently, several projects in China started to investigate how modern measurement and information and communication technologies can support not only exact estimation of the meridian status, but also to improve TCM in general [13. Although Chinese and Western medicine have a different understanding and approach to life, health, and illness - concerted, complementary work between Western allopathy and Chinese medicine could result in an improved health system [14. The problem of diabetis mellitus [15] is urgent around the world (estimated 100 million [16]), in Austria approximately 300.000 people suffer from it [17. To minimize short term problems (e.g. unconsciousness, hypoglycemia) and severe mid and long term complications (e.g. blindness and neuropathie), which are also very costly to treat, repetitive invasive blood sugar measurements are needed with following medication modulation (typically insulin for Type 1 and pills for Type 2 diabetics). Besides several negative medical side effects (e.g. skin injuring), this way is also rather expensive.

This paper proposes the grid based implementation of a novel non-invasive method for measuring human blood glucose values accurately and conveniently by the use of a special medical meridian measurement instrument and the CADGrid infrastructure [18. The data obtained by this instrument are referred to as meridian measurement data and can be analyzed by the meridian electro information transmission model [19] to derive human blood glucose values. This model is implemented as a complex grid-based computing process that executes a number of compute intensive algorithms. The controlled execution of this process is done by the Non-Invasive Glucose Measurement Service, in short NIGM-Service. A special subset of CADGrid-services will focus on receiving and analyzing patients meridian measurement data coming from mobile devices and thin clients. Splitting the process of vital parameter estimation into an evolveable, personalized data mining process and a rather simple source signal emitting and recording phase has two main benefits: first, the infrastructure can easily be applied to other target values and second, improvements to the involved data mining services and process will directly result in a more precise and robust estimation of the values. Diabetic patients as well as researchers in the field of treatment of diabetes mellitus will highly profit from the realization of the above mentioned scientific computing infrastructure.

This paper is organized as follows. In Section 2 the application domain is described in order to provide the reader with domain specific background knowledge. Section 3 describes the China-Austria Data Grid infrastructure and 
on top of it its first application the Non-Invasive Glucose Measurement Service in Section 4. Section 5 gives a brief overview of available non-invasive methods for measuring human blood glucose values. Finally, we conclude the paper and mention our ongoing work as well as planned next steps in Section 6 .

\section{Application Scenario}

The non-invasive method for measuring human glucose values used in the NIGMService is based on the meridian-theory, according to which the human body has 14 acupuncture meridians. Each of these longitudinally distributed lines on our human body has its main points, called source points, totally 24 [20]. Clinical practices of TCM (especially acupuncture) have been guided by meridian theory for thousands of years.

Exact estimation of the meridian status has been one of the pilot tasks of TCM. In order to prove the meridian theory with modern methods a number of special meridian measurement instruments were developed. Analyzing these meridian measurement data with advanced data mining techniques and models can lead to important information about human illness state and other health relevant knowledge.

The electro signal measurement instrument sends an electric signal (white noise) into one meridian source point and measures the corresponding signal output at another source point either on the same meridian or on another meridian. In particular a random electro signal with the maximal voltage less than $2.0 \mathrm{~V}$ is produced by the instrument. This process is illustrated in Figure 1. According to international standard terminologies defined by the World Health Organization on traditional medicine in the western pacific region [21, point 1 is called "Ba Xie" and point 2 is called "He Gu". These points are connecting two different meridians through a special information channel. Zero potential points are points on the human body that have the lowest electrical potential and are usually located on finger tips and toes. In the context of electro signal measure, zero point measures are used as reference values, in order to weight the electric potential on a acupuncture point. The measurements obtained in this process can, if analyzed by the meridian electro information transmission model, derive diabetic patient's blood glucose values.

Another instrument measures the subcutaneous impedance at acupuncture points and its adjacent control points 20. Also high sensitive $\mathrm{CO}_{2}$ analyzers are used to measure transcutaneous $\mathrm{CO}_{2}$ emission 22 from the skin on correlating meridian source points. Using clustering and frequent itemset mining techniques, correlations among measured values of these points can be identified, e.g. start-end point correlation, symmetric point correlation etc., which is useful knowledge for research in the meridian theory and thus aims to observe the features of acupuncture and meridians. Deploying an e-infrastructure that provides collaborative research with advanced data mining services, efficient data and workflow management services, as well as visualization services contribute to the progress in this domain. 


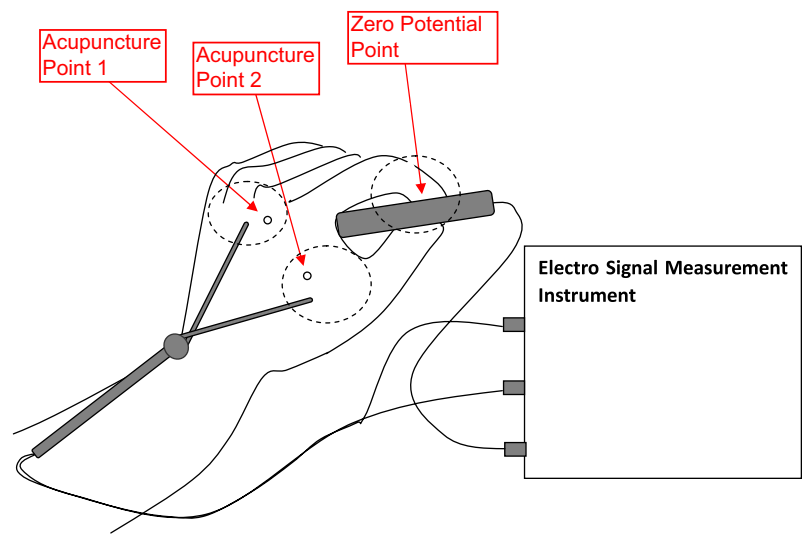

Fig. 1. The Electro Signal Measure on the left hand

\section{CADGrid Infrastructure}

Science has evolved in the past several decades from an empirical and theoretical approach to one that includes computational simulations and modeling [23], commonly known as enhanced science or e-science in short. The term e-Science is often used to refer to highly collaborative computational science that uses distributed software infrastructures, like grids in order to support shared efforts. Technological progress in such e-Science infrastructures have enabled researchers to run complex, computational investigations that include data access, analysis, and largely automated model execution.

The China-Austria Data Grid (CADGrid) connects several research institutions in both countries and provides an Intelligence Base offering commonly used models and algorithms as services as well as compute and storage resources. Further it is equipped with our workflow engine WEEP [24] allowing the users to execute a number of pre-selected services in a controlled and efficient way.

The Intelligence Base is the heart of the CADGrid infrastructure, providing participants a number of services aiming to support and accomplish user defined research tasks. In general the services can be classified into four main groups, (a) data analysis group includes services representing data mining and other special data analysis algorithms (i.e. Kalman Filtering), (b) data managing and preprocessing services covering issues that occur when working with different data sources or when accessing data resources from different locations, (c) workflow services that are necessary to provide the controlled execution of a number of pre-selected services, and (d) the data visualization group including services for data representation and visualization.

Three typical usage scenarios will exemplify how diabetic patients, medical professionals, and researchers in the field of treatment of diabetes mellitus can benefit from NIGM and its underlying grid infrastructure. 
Diabetic patients can measure the electricity information transformation of their blood sugar values using the measurement instrument which will be embedded into mobile devices such as PDA, cell phones and other handhelds. Using the NIGM-Service offered via the Intelligence Base the measurement data can be analyzed on the fly and the patient will be informed via messaging services about his blood glucose values. Patients will benefit twice: first the incurred expenses will be lower in comparison to conventional methods based on chemical reactions and second patients will have a much more convenient method than injuring their skin to receive the needed drop of blood for the chemical reaction.

Health professionals and services have a better surveillance of the patient's illness and can adjust their therapy more efficiently based on the continuously collected data.

Scientists from different institutions in different countries can work together on common goals. This will require access to anonymized measurement data and analysis services from different locations. Further they might want to share their results among each other. The CADGrid Intelligence Base provides advanced data management services enabling such requirements in an efficient and secure way. By means of the online portals, scientists can access advanced data analysis tools, computational power, and data resources and share elaborated results.

\section{Non-Invasive Glucose Measurement Service}

The NIGM-Service covers the process of computing patients glucose values from meridian measurements. The service consists of the execution of several algorithms, each one available as a standalone CADGrid service within the Intelligence Base. Using the workflow engine WEEP [24, a predefined workflow representing the NIGM-Service is deployed and available as one service within the CADGrid Intelligence Base. Figure 2 illustrates the workflow with the visualized outputs of each component.

In the following the non-invasive process of calculating patients blood glucose values from meridian measurements is briefly described. More details can be found in [19]:

Collect Measurement Data by running a measurement process with the electro signal measurement instrument. This non-invasive painless process is done by a health professional or any trained person on the hand of a patient. The current prototype of the instrument is equipped with a USB interface and connected to a PC, where the occurred measurement data is organized in two files. One representing the input values and the other contains values of the measured output signal. This data, referred to as meridian measurement data is sent to the joint grid testbed where the NIGM-Service will be started. The NIGM-Service is composed of a set of services implementing the following algorithms, which will be executed one after the other, whereas each algorithm's output represents the input to the next algorithm.

System Identification is the first algorithm to be executed. A model is set up for each measured input/output value pair describing the relationship 


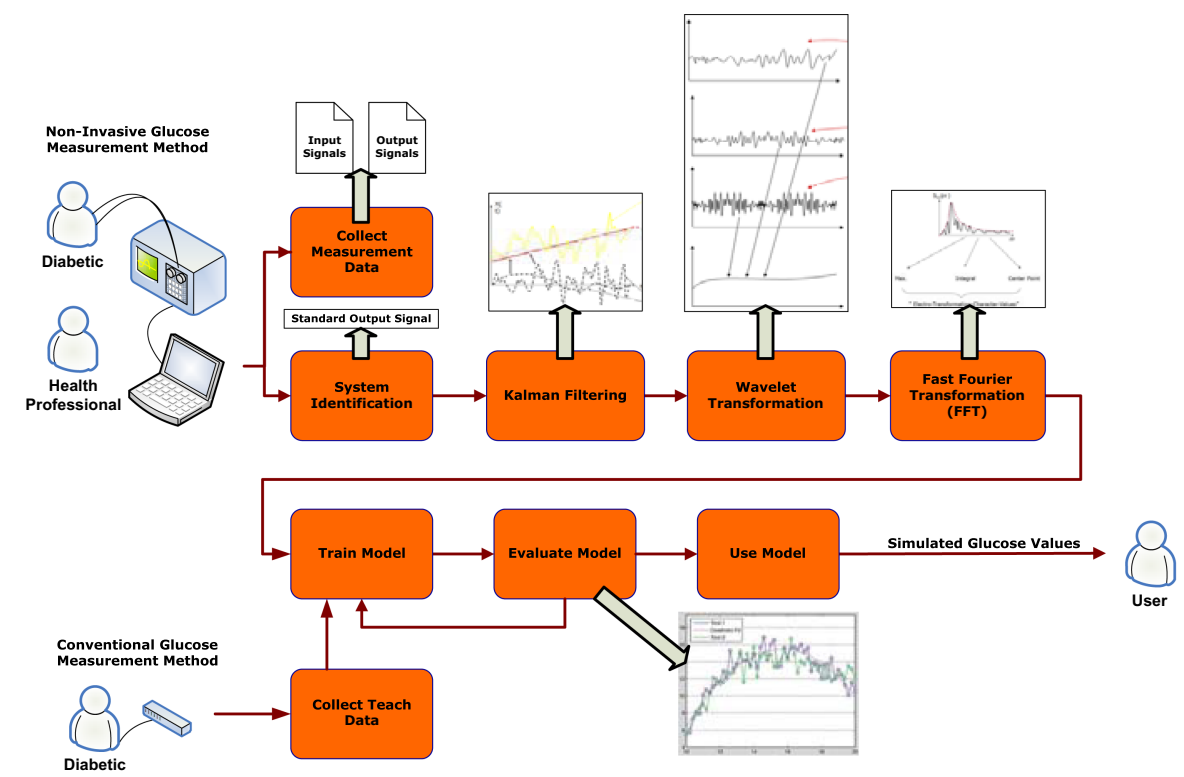

Fig. 2. The Non-Invasive Glucose Measurement Service

between the corresponding signals. The resulting difference equation is used to generate a standard output signal for a common input signal.

Kalman Filtering is further used to weaken the white noises and filter the useful signals, as well as useful noises. In fact, the useful signals are the true data retrieved from the meridians. However, the measurement data is blended with different kind of noises: physical, chemical, and biological noises. These noises can represent the patient's condition and feelings at the moment the measurements were taken i.e. fear, hotness, etc. and also the surroundings of the patient i.e. noisy or electromagnetic environment. The first kind of noises represent useful information (color noise), whereas the latter one (white noise) are of less interest. Although they need to be extracted in order to get data that is close to the true data. This process is defined as optimal estimation of measured true data, which is blended by white and color noises and is covered within the Kalman Filtering service.

Wavelet Transformation takes the graph containing the optimally estimated data produced in the previous step and splits it into several frequency levels. Each generated new graph, referred to as wavelet represents the origin time domain graph from different frequency levels. This mechanism of experimental data processing provides the most visual and informative picture of the measured data and allows finding peculiarities of the signal in the area of the wavelet. Hence the wavelet with the most important graph is selected and forwarded to the next algorithm. It is to say that choosing a best wavelet for calculating the blood glucose values is in the current state of research progress an optional step and needs to be elaborated in detail. 
Fast Fourier Transformation is then executed in order to produce the frequency domain representation of the time domain graph used in the previous algorithms. A frequency domain graph shows how much of the signal lies within each given frequency band over a range of frequencies. Further the so called electro-transformation-character-values are computed. These values, such as maximum, integral, center point, etc. represent the characteristics of the produced graph. These are the main inputs for the next step which is to setup a back propagation neural network.

Back Propagation Neural Network. In general such a model is used to establish a mathematical model describing the relationship among two data series. The two data series in our model are the electro-transformation-character-values on one side and conventionally measured glucose values, serving as training data in order to set up the model. The model has to be trained first, pictured in the left corner of the figure above. During this phase, in addition to each meridian measurement a conventional method has to be used to get reference values. Once a model is set up for a patient it can be used to compute the patient's glucose values for a given set of electro-transformation-character-values without conventionally measured values. In order to maintain the model, reference values have to be measured again in a certain time interval (e.g. once a week) and the model must be re-evaluated to accommodate recent changes and developments in the patient health condition and to further improve precision and robustness.

\section{Related Work}

The development of non-invasive methods for measuring human blood glucose values has been targeted by a number of research institutions all over the world. The most common non-invasive methods were categorized into two broad categories [16]: (a) blood glucose measurement with external factor and (b) without external factor (based on the theory that blood glucose values can be derived from glucose concentration in human body liquids like tears, urine and saliva). The latter cannot be used for continuous measurement of glucose concentration in blood. Methods with external factor are described in the following:

Electromagnetic Radiation. Here electromagnetic radiation (EMR) is used as an external factor for the non-invasive measurement of glucose value in blood. The optical characteristics of the tissue are observed and analyzed when a human body is exposed to EMR. Experiments have shown that optical methods are not sufficient to calculate blood glucose values accurately, therefore other external factor techniques like mechanical stimulation of tissues and analysis of light scattering are more frequently used.

Eye Sensitivity to small color changes. In this technique the value of glucose in blood is measured from the eye sensitivity to small color changes where wide range of wavelengths, filters and detectors are used.

Endogenous Method. This method correlates changes in the glucose value to changes in the electro-physiological activity of certain peripheral nerves. 
Changes in their endogenous physiological and biochemical functions are observed from which the glucose value is calculated.

Nuclear Magnetic Resonance. In this method the nuclear magnetic resonance spectrum and water resonance are considered. Blood glucose values are calculated on the basis of theory that the ratio of the resonance frequencies correlates with the blood glucose level.

Our approach has the advantage to split the process of blood glucose value estimation into a developable, personalized data mining process and a rather simple source signal emitting and recording phase. Improvements to the involved data mining services and overall process can be deployed remotely and will directly result in a more precise and robust estimation for the patient without the need to change the client environment. Another important benefit of our approach is that the introduced concept of local measurement and remote personalized estimation is general applicable to various scenarios, e.g. for estimating another vital parameter like blood pressure.

E-Health 25], the use of information and communication technologies to improve or enable health care, will have various impacts on health care services [26] and the quality, cost and efficiency of patient care [27. Health Grids [27] are going to be used for the individualized health care and the epidemiology analysis. The former is improved by the secure and efficient combination of the widespread personal clinical information and the availability of advanced services for diagnostic and therapy. The latter combine the information from a wide population to extract the knowledge that can lead to the discovery of new correlations between symptoms, diseases, genetic features or any other clinical data. An example of a project serving both directions is @neurIST [3], which aims to create an IT infrastructure for the management of all processes linked to research, diagnosis and treatment development for complex and multi-factorial diseases - currently focusing on cerebral aneurysm and subarachnoid haemorrhage.

The recently started EU project OLDES 28 includes a pilot application focusing on older persons suffering from Type 2 diabetes mellitus. The main aim is to provide a low-cost and easy-to-use health care platform, including a continuous glucose monitoring system. All measured data, including other vital parameters, will be transferred wirelessly to the patient's health provider. This will enable a better surveillance of the patient's health status and more efficient adjustment of therapy. While we are having the same overall aims as OLDES namely the reduction of acute and chronic complications of diabetes and a more effective prevention of emergencies, resulting in the reduction of the frequency of hospitalizations and increased quality of life - we are targeting also the commonly younger, more mobile and active Type 1 diabetics with our non-invasive measurement method, but not providing such a comprehensive health platform.

\section{Conclusions}

This paper has outlined the China-Austria Data Grid framework in the context of its first application, namely the Non-Invasive Glucose Measurement Service, 
in short NIGM-Service. The key contribution in this paper is the grid based implementation of a novel non-invasive method for accurate estimation of blood glucose values based on electro-transformation measures in human body meridians. The presented approach has two main benefits, by splitting the process of vital parameter estimation into an evolve-able, personalized data mining process and a rather simple source signal emitting and recording phase: first, the infrastructure can easily be applied to other target values and second, improvements to the involved data mining services and process will directly result in a more precise and robust estimation of the values. It follows from the discussion in this paper that the treatment of diabetic patients will be the first domain highly profiting (improvement of quality of life, economical aspects, etc.) from the NIGM-Service. However, the scientific computing infrastructure presented in this paper also establishes the basis for a number of future applications and extensions to other domains.

Acknowledgments. The work described in this paper is being carried out as part of the research projects "Medical Measurement Grid for On-Line Diagnosis" and "Austrian Grid" supported by the Austrian Federal Ministry of Science and Research.

\section{References}

1. De Roure, D., Baker, M.A., Jennings, N.R., Shadbolt, N.R.: The evolution of the grid. In: Berman, F., Hey, A.J.G., Fox, G. (eds.) Grid Computing: Making The Global Infrastructure a Reality, pp. 65-100. John Wiley \& Sons, Chichester (2003)

2. Ramakrishnan, L., Simmhan, Y., Plale, B.: Realization of Dynamically Adaptive Weather Analysis and Forecasting in LEAD: Four Years Down the Road. In: Shi, Y., van Albada, G.D., Dongarra, J., Sloot, P.M.A. (eds.) ICCS 2007. LNCS, vol. 4487, pp. 1122-1129. Springer, Heidelberg (2007)

3. The @neurIST Project: Integrated Biomedical Informatics for the Management of Cerebral Aneurysms (2008), http://www.aneurist.org/

4. Benkner, S., Berti, G., Engelbrecht, G., Fingberg, J., Kohring, G., Middleton, S.E., Schmidt, R.: GEMSS: Grid-infrastructure for Medical Service Provision (2004)

5. Krishnan, A.: A survey of life sciences applications on the grid. New Generation Computing 22(2), 111-126 (2004)

6. Srinivasan, L., Treadwell, J.: An overview of service-oriented architecture, web services and grid computing. Hewlett-Packard White Paper (2005)

7. Abowd, G.D., Mynatt, E.D.: Charting past, present, and future research in ubiquitous computing. ACM Transactions on Computer-Human Interaction (TOCHI) 7(1) (2000)

8. Satyanarayanan, M.: Fundamental challenges in mobile computing. In: PODC 1996: Proceedings of the fifteenth annual ACM symposium on Principles of distributed computing, pp. 1-7 (1996)

9. Mattern, F.: Ubiquitous computing: Scenarios from an informatised world. In: Zerdick, A., et al. (eds.) E-Merging Media - Communication and the Media Economy of the Future, pp. 145-163. Springer, Heidelberg (2005)

10. The AKOGRIMO Project: Access to Knowledge trough the Grid in a Mobile World (2008), http://www.mobilegrids.org/ 
11. Cannataro, M., Talia, D.: Towards the next-generation grid: a pervasive environment for knowledge-based computing. Information Technology: Coding and Computing, 437-441 (2003)

12. Maciocia, G.: The Foundations of Chinese Medicine: A Comprehensive Text for Acupuncturists and Medical Herbalist. Elsevier Churchill Livingstone (2005)

13. Wen, D.: The drum of chinese medicine's modernization beats loudly: A summary of the second international science and technology conference on traditional chinese medicine. Acupuncture Today (2005)

14. Schloegl, R.: Report on the outcome of the conference 'traditional chinese medicine - a successful concept for east and west'. In: Seminar on the modernization of traditional medicine, pp. 10-11 (2006)

15. WHO-IDF: Definition and diagnosis of diabetes mellitus and intermediate hyperglycemia. Report of a WHO/IDF Consultation (2006)

16. Bazaev, N.A., Selishchev, S.V.: Noninvasive methods for blood glucose measurement. Biomedical Engineering 41(1), 42-50 (2007)

17. Diabetes-Austria (2008), http://www.diabetes-austria.com/

18. CADGrid: China-austria data grid (2007), http://www.par.univie.ac.at/project/cadgrid/

19. Han, J., Han, Y., Xia, Q., Hou, X., Li, Y.: Experiment and analysis on transmission-characteristics of human-body-meridians-electro-information with application. Modern Chinese- and west-Medicine Magazine 3(19), 17-29 (2005)

20. Zhang, W.B., Jeong, D.: Subcutaneous impedance measured by four-electrode method and low impedance acupoints located by single power alternative current. American Journal of Chinese Medicine 32(5), 779-788 (2004)

21. WHO: International standard terminologies on traditional medicine in the western pacific region. WHO Library Cataloging in Publication Data (2007)

22. Zhang, W.B.: Proceedings in the study of transcutaneous CO2 emission in acupuncture and meridians. World Journal of Gastroenterol 6(3) (2000)

23. Bell, G., Gray, J., Szalay, A.: Petascale computational systems. Computer 39(1), 110-112 (2006)

24. WEEP: The workflow enactment engine project (2005), http://weep.gridminer.org

25. Oh, H., Rizo, C., Enkin, M., Jadad, A.: What is ehealth (3): A systematic review of published definitions. Journal of Medical Internet Research (2005)

26. Martin, S., Yen, D.C., Tan, J.K.: E-health: impacts of internet technologies on various healthcare and services sectors. International Journal of Healthcare Technology and Management 4(1/2), 71-86 (2002)

27. Breton, V., Dean, K., Solomonides, T.: The healthgrid white paper (2005), http://www.whitepaper.healthgrid.org

28. The OLDES project: Old people's e-services at home (2007), http://www.oldes.eu/ 